\title{
The Solar Chemical Composition: Past and Present
}

\author{
Nicolas Grevesse $e^{1,2}$ \\ ${ }^{1}$ Centre Spatial de Liège, Université de Liège, avenue Pré Aily, B-4031 Angleur-Liège, Belgium \\ ${ }^{2}$ Space sciences, Technologies and Astrophysics Research (STAR) Institute, \\ Université de Liège, 19c allée du Six Août, B-4000 Liège, Belgium
}

\begin{abstract}
We briefly review the solar chemical composition derived last century and the new, different solar chemical composition, obtained since about 15 years.
\end{abstract}

Keywords: solar abundances - solar photosphere

\section{Introduction}

We briefly tell the history of the different determinations of the solar chemical composition, essentially based on photospheric spectra, since about 40 years, first the old solar abundances derived with 1D models of the photosphere together with the hypothesis of LTE. We then turn to the new way we use nowadays i.e. realistic 3D models and non-LTE calculations. The present day results lead to a decrease of the abundances of the most abundant metals, $\mathrm{O}, \mathrm{C}$ and $\mathrm{N}$. We then compare the old and the new results and discuss why the abundances of these elements have decreased. We take the example of oxygen as it is the largest contributor to $\mathrm{Z}$, the metallicity.

We then briefly discuss the impacts of the present chemical mixture, emphasizing the so-called "solar modeling problem": the standard solar models computed with the new abundances disagree with the observations derived from helioseismology whereas a very good agreement was obtained during quite a long period of time when using the old solar chemical composition. We conclude with the most recent photospheric abundance results obtained when using the modern technique, 3D models and precise non-LTE.

\section{The Past}

The early beginnings of the researches on the solar chemical composition, since Russell in 1929, have been summarized by Grevesse and Sauval (1998); they will not be repeated here. During the last two decades or so of the last century, various new solar chemical compositions have been published (see e.g. Anders \& Grevesse 1989; Grevesse \& Noels 1993 and Grevesse \& Sauval 1998; these works are often abbreviated as AG89, GN93 and GS98 respectively). All these new analyses used, on the one hand, 1D models of the solar photosphere together with the local thermodynamic equilibrium (LTE) hypothesis and, on the other hand, solar data, i.e. spectra, covering the visible up to the far IR, that are 
essentially the same as the ones used today. The well-known metallicity, Z, slowly varied from 0.02 to 0.018 . These changes on the solar composition were essentially due to progress in the accuracy of the atomic and molecular data (the transition probabilities) used to derive the abundances.

\section{The Present}

\subsection{D Models}

Since the beginning of this century, the way we derive solar abundances has completely changed. This is not an evolution but a revolution! The 1D photospheric models previously used for many decades have been replaced by 3D models. The main characteristics of the 3D models, calculated with the Stagger code (see Stein and Nordlund ,1998 and Nordlund et al., 2009, and references therein) are the following:

- These 3D models really outperform all the 1D models used.

- These models are highly realistic, reproducing successfully a wide range of observational constraints.

For the first time, the observed heterogeneous and ever changing nature of the photosphere (granulation) is successfully modeled. Moreover and for the first time also, the 3D models allow to reproduce the solar line profiles as they are really observed in the solar spectrum i.e.

- all the line profiles are asymmetric,

- the bisectors of the unblended lines show a delicate C-shape,

- the wavelength of the centers of the lines are shifted to the blue.

These subtle characteristics in the shapes of the lines result from the complex motions of matter in the photosphere. These material motions themselves result from subtle overshooting of matter at the upper limit of the solar convection zone and propagating to high photospheric layers. Obviously, 1D models cannot reproduce these subtle but important properties of the line profiles, leading to unshifted, symmetric lines. Furthermore, the widths of the spectral lines are predicted naturally with the 3D models without invoking any fudge parameter like micro- and macro-turbulence needed with 1D models.

In addition, the new generation of 3D models reproduce in detail the observed center-to-limb variation of the continuous intensity as well as the absolute intensity. 3D models are undoubtedly a great step forward in our modeling of the real nature of the solar photosphere.

\subsection{Non-LTE Analyses}

In addition, the often used LTE hypothesis has been replaced by non-LTE analyses that are now becoming more accurate. Realistic non-LTE analyses need to rest on a model atom/ion as complete as possible. This requires on the one hand to know quite a large number of accurate transition probabilities and, on the other hand, to know the cross sections for the collisions with the electrons (rather well-known) as well as the cross sections for the collisions with the neutral hydrogen atoms. These last data were very approximate last century; thus reliable non-LTE could not be done. Since about 
15 years such data become slowly available for a larger number of elements and we can thus today make reliable non-LTE analyses.

\subsection{The Solar Abundance of Oxygen}

The abundances of all the elements present in the Sun have been derived by Asplund et al. (2005) and Asplund et al. (2009, AGSS09), using the new techniques just described. In the present paper we shall essentially discuss the problem of the decrease of the abundances of $\mathrm{C}, \mathrm{N}$ and $\mathrm{O}$, from AG89, GN93 and GS98 to AGSS09. We shall insist on the case of oxygen which is by far the dominant contributor to Z, the metallicity.

\subsubsection{The Indicators of Oxygen Abundance}

The available indicators of the abundance of oxygen are three forbidden lines of OI with very low excitation energies, a few very high excitation (larger than $9 \mathrm{eV}$ ) permitted OI lines and a very large number of vibration-rotation and pure rotation lines of $\mathrm{OH}$ in the IR, very clean and easy to measure with accuracy. It is easy to show that, at solar photospheric temperatures, most of the $\mathrm{O}$ is in the lower OI levels. That means that the forbidden lines are not sensitive to departures from LTE and not very sensitive to the temperature i.e. the model. The very high excitation permitted OI lines, with level population of about $10^{-9}$ compared with the ground levels, are on the contrary extremely sensitive to departures from LTE. It can also be easily shown that the molecular lines are not more sensitive to the temperature than the high excitation OI lines, the only difference is that they are formed in somewhat higher layers than the atomic lines. Furthermore, it is also easy to show that you have many orders of magnitude more $\mathrm{O}$ in $\mathrm{OH}$ than in the lower levels that produce the high excitation permitted lines of OI.

To summarize, the best indicators of the $\mathrm{O}$ abundance are the low excitation forbidden lines (unfortunately blended, see Sect. 3.3.2), followed by the molecular lines of $\mathrm{OH}$ easily measured on the IR solar spectrum, and finally, the very high excitation lines of OI, used by many authors, but very sensitive to the model and more to the non-LTE effect.

\subsubsection{Differences between GN93 and AGSS09}

The abundance derived from the three forbidden lines of OI by GN93 is high, A(O)=8.95 (the abundances are given in the usual logarithmic scale relative to hydrogen where $\log N(H)=12$ ). In the meantime it has been discovered that each of these ideal indicators of the $\mathrm{O}$ abundance is unfortunately blended. AGSS09 took the blends very carefully into account, in a purely empirical way, independent of any atmospheric model; this led to a decrease of the $\mathrm{O}$ abundance down to 8.69. The impact of the use of the 3D model rather than the 1D model atmosphere is negligible for these forbidden lines.

The handful of high excitation OI lines were treated in LTE by GN93 although we already suspected possible departures from LTE. But the required atomic data to compute the lines in non-LTE were not available. The result of GN93 is an abundance of $\mathrm{O}$ of 8.83, somewhat lower than the value obtained from the forbidden lines. AGSS09 have been able to treat the non-LTE excitation of the OI lines with great accuracy. They have been able to calibrate one of the poorly known data in the nonLTE calculations, namely the cross-sections for the collisions with the very abundant neutral hydrogen atoms in the photospheric layers. They calibrated these cross-sections from very precise center to limb observations of a few of these high excitation OI lines. The very large non-LTE corrections to 
the LTE abundances are negative and the AGSS09 oxygen abundance value decreased to $A(O)=8.69$. This last value has very recently been confirmed by Amarsi et al. (2018) from new very accurate 3D non-LTE calculations applied to these high excitation lines as well as to center to limb observations of these lines. The impact of the new 3D model is also very small.

The only indicators that are very sensitive to the use of a 3D rather than a $1 \mathrm{D}$ model atmosphere are the molecules. The mean temperature of the 3D model in the outer layers where the molecular lines are formed appears to be somewhat smaller than the corresponding temperature in the 1D models. Therefore, the 3D AGSS09 result from the OH lines is smaller (8.69) than the 1D value of GN93 (8.87).

Let us summarize the differences New-Old for oxygen: $A(O)=8.69$ (AGSS09) versus 8.87 (mean value of GN93).

- The decrease of the abundances from the forbidden lines is due to the presence of blends unknown 15 years ago.

- The decrease of the abundance from the high excitation permitted OI lines is due to the departures from LTE affecting those lines, effects that could not be computed 15 years ago because of the lack of the required atomic data.

- The decrease of the abundance from the molecular lines is really the result of the new 3D model.

The new final abundances from the 3 different indicators are now in perfect agreement. The old values, for the three indicators, also agree by chance but with much larger differences between the various indicators.

\subsection{The Results from AGSS09}

Applying the new techniques, 3D models and non-LTE when possible, AGSS09 redetermined the abundances of all the elements present in the sun. For carbon, we have a situation very similar to oxygen. For the same reasons as for $\mathrm{O}$, the abundance of $\mathrm{C}$ has decreased by 0.12 dex from GN93 to AGSS09. The abundance of nitrogen has also decreased by 0.09 dex. Their results do thus show a serious decrease in the abundances of $\mathrm{C}, \mathrm{N}$ and $\mathrm{O}$ just mentioned.

For the heavier elements, much less important changes are observed between old and new values: see e.g. Scott et al. (2015a, 2015b) and Grevesse et al. (2015) as well as very recent new results for a few elements obtained thanks to refined non-LTE analyses mentioned in Sect. 6. These new results imply that the metallicity, $\mathrm{Z}$, dropped from about 0.02 down to 0.0134 as $\mathrm{O}$ and $\mathrm{C}$ are main contributors to Z (O: $42 \%$, C: $18 \%$; see also Sect. 6).

\subsection{Comparison with Meteorites}

As already discussed by AGSS09, the new photospheric abundances (see also Sect. 6) agree rather nicely with the meteoritic abundances (see Table 1 where the meteoritic data are mean values between Lodders et al. (2009) and Barrat et al. (2012)). However, when examined very carefully, there is a slight but real dependence of the differences sun-meteorites versus condensation temperature (M. Asplund, private communication). CI meteorites were believed to be the least fractionated meteorites, 
and represent the original solar system material. There are now good reasons to doubt that CI chondrites are samples of mean solar system materials ( $\mathrm{J}$ Wasson, private communication). So, even if they now agree with the phosphoric value, the very precise meteoritic abundances cannot be used as substitutes of higher precision to the photospheric abundances.

\subsection{Differences between recent solar mixtures}

Caffau et al. (2011 and references therein) have also revisited the solar abundances of a few elements using their own 3D model of the solar photosphere. The abundance results from these authors are slightly larger than AGSS09 : for example $\mathrm{A}(\mathrm{O})=8.76$ compared with 8.69 (AGSS09) and $\mathrm{A}(\mathrm{C})=$ 8.50 compared with 8.43 (AGSS09).

We have been able to identify the main reasons for these differences that are not at all related to the use of different 3D models. These models agree and the differences in abundance results are always lower than about 5\%. If we examine the oxygen results, only based on the atomic lines in Caffau et al. (2011), we see that their somewhat larger values from the forbidden lines should decrease if the blends were more accurately estimated, the value from the permitted lines could also somewhat decrease if their solar data as well as the non-LTE results were updated.

For carbon, the very large dispersion in their results ( \pm 0.11 with a difference of 0.48 dex, a factor 3 , between their highest and smallest result) is clearly produced by their use of many strong CI lines that are slightly blended leading to the somewhat too large abundance result mentioned here above.

\section{Impact of our New Chemical Composition on the Solar Model}

Standard solar models (SSM) constrained to fulfill GS98 (high-Z) or AGSS09 (low-Z) compositions show quite different internal structures. In particular, solar models based on the AGSS09 composition show strong discrepancies with helioseismic probes of the solar interior, whereas the older GS98 composition leads to quite nice agreement (Christensen-Dalsgaard 2002, Basu \& Antia 2004, Serenelli et al. 2009, Vinyoles et al. 2017, among many). This discrepancy is known as the "solar modeling problem". The seismic probes relevant to the solar modeling problem include the profile of the solar sound speed, the depth of the convective envelope, the surface helium abundance and frequency separation ratios.

This solar modeling problem has been around for more than 10 years since the first donward revision of the $\mathrm{O}$ and $\mathrm{C}$ abundances by Allende Prieto et al. (2001, 2002) and Asplund et al. (2004, 2005). While there is no lack of proposed ideas, no definitive solution has yet been found. It is well known that the comprehension of the solar abundance problem is intimately related to understanding the role of opacity in solar modeling. For a more detailed account of these problems and possible solutions, see also Buldgen et al. (2018 and references therein) as well as J. Montalbán and also G. Buldgen, this volume.

\subsection{The problem of the Solar Opacity}

The effects produced by a modification of the radiative opacity are almost degenerate (with the notable exception of CNO neutrinos) with those produced by a modification of the heavy element admixture. Namely, the agreement with helioseismology using the AGSS09 composition could be restored by a suitable modification of the opacity profile of the Sun (about $15 \%$ at the basis of the convection zone 
and much less, about 3\% at the center of the sun) as described in Christensen-Dalsgaard et al. (2009), Serenelli et al. (2009), Villante (2010) and Villante et al. (2014). The current generation of standard solar models typically relies on opacities from the Opacity Project (OP, e.g. Badnell et al. 2005) or OPAL (e.g. Iglesias \& Rogers 1996). The contributions of different chemical elements in the inner solar layers are well illustrated by Blancard et al. (2012, see their figure 2). The above mentioned opacity tables are based on theoretical calculations.

The opacity due to iron at the basis of the convection zone (CZ; Fe contributes to about $20 \%$ to the opacity) has been and is still analyzed by A. Pradhan (private communication). Each time he progressed in the completeness of Fe XVII, the most abundant Fe ion in these layers, the opacity increases. Recently, and for the first time, Bailey et al. (2015) experimentally measured the opacity due to Fe at conditions similar to those immediately below the solar convection zone, finding wavelengthdependent Fe opacities typically 30 to $40 \%$ higher than predicted by OP and OPAL. When folded into the Rosseland mean opacity needed in solar model calculations, the result is a $7.3 \%$ increase. Such extra opacity goes towards solving the solar modelling problem by itself. Recently, also, theoretical work by Krief et al. (2016) found that line broadening induces increases in the opacity that mimics the opacity variations required by helioseismic constraints. From the experimental result and from his own theoretical results, A. Pradhan (private communication) suggested that the present day used opacities were underestimated, with potential increases larger at the basis of the $\mathrm{CZ}$ than at the center of the sun. Detailed solar modelling however will have to await a better understanding of how such opacity increases depend on the physical conditions (temperature and density) for the elements that contribute mostly to the opacity, O, Ne, Fe essentially (see also S. Salmon, this volume for a more complete review).

\section{A Special Case: Solar Neon}

As neon does not show any line in the photospheric spectrum, the abundance of neon is derived from the analysis of coronal spectra and from measurement in the solar wind and solar energetic particles. As it is very difficult to derive directly the $\mathrm{Ne} / \mathrm{H}$ ratio, the abundance of $\mathrm{Ne}$ is derived from the ratio $\mathrm{Ne} / \mathrm{O}$. Unfortunately, this ratio has been observed to vary depending on the solar matter used, on the solar wind speed and also on the solar cycle. The solution suggested by Young (2005a, 2005b) is to use quiet solar matter. From XUV solar spectra of quiet matter he found $\mathrm{Ne} / \mathrm{O}=0.175$. We adopted this value in AGSS09. And, since $\mathrm{O}$ has decreased by about 0.2 dex when compared with older abundances, the Ne abundance reported by AGSS09 also decreased by the same amount. Very recently however Young (2018) revised his analysis of 2005 with new better atomic data and found $\mathrm{Ne} / \mathrm{O}=0.244 \pm 0.050$. Then, with our low abundance of $\mathrm{O}, \mathrm{A}(\mathrm{O})=8.69$, the present solar abundance of $\mathrm{Ne}$ is $\mathrm{A}(\mathrm{Ne})=8.08 \pm 0.09$, by chance about the same value that was adopted in the older solar abundance tables.

\section{Very Recent non-LTE Results}

Very recently, Amarsi et al. (2018) revised the oxygen abundance derived from the most important O I lines observed at the center but also at different positions on the solar disc. Their analysis uses refined 3D non-LTE calculations and their result is $\mathrm{A}(\mathrm{O})=8.69$, confirming the value from AGSS09. For carbon, Amarsi et al. (2019) also revised the abundance derived from C I lines using 3D models together with a sophisticated 3D non-LTE analysis. They found $\mathrm{A}(\mathrm{C})=8.44$, only 0.01 dex larger than the value of AGSS09. The abundances of all the elements heavier than Na have been revised by Scott 
et al. (2015a, 2015b) and Grevesse et al. (2015), using 3D models and non-LTE when possible. New very accurate non-LTE analyses have also recently been made by Lind et al. (2017, Fe), Bergemann et al. (2017, Mg), Amarsi and Asplund (2017, Si), Nordlander \& Lind (2017, Al), Osorio et al. (2019, Ca), Carlos et al. (2019, Li), Reggiani et al. (2019, K).

All these new results are collected in Table 1 (NEW) where they are compared with the AGSS09 results as well as with the meteoritic data. The more precise results of these new analyses show very small differences, of the of order 0.01 to 0.04 dex, with those of AGSS09 except for Ne that has increased by $40 \%$ ( $0.15 \mathrm{dex})$ as mentioned in Sect. 5 . In addition, the new results also confirm the small uncertainties of the order of 0.04 dex for the most important elements.

With these new data, we find $\mathrm{X}=0.7362, \mathrm{Y}=0.2500$ and the metallicity $\mathrm{Z}=0.0138(\mathrm{Z} / \mathrm{X}=$ $0.0187)$, with an uncertainty of $8 \%(0.0011)$. This last value is in very good agreement with the low $\mathrm{Z}$ already found by AGSS09, $\mathrm{Z}=0.0134$ (with $\mathrm{X}=0.7381$ and $\mathrm{Y}=0.2485$ ). Because of its increased abundance, Neon becomes the third contributor to the metallicity: $\mathrm{O}(41.7 \%)$ followed by $\mathrm{C}(17.6 \%)$ and $\mathrm{Ne}(12.9 \%)$ and then $\mathrm{Fe}(9 \%), \mathrm{N}(5 \%), \mathrm{Si}(4.8 \%), \mathrm{Mg}(4.7 \%)$ and $\mathrm{S}(2.2 \%)$. We can also derive a protosolar chemical composition of $\mathrm{X}=0.7145, \mathrm{Y}=0.2710$ and $\mathrm{Z}=0.0146$, as done by AGSS09 (their Sect. 3.11 and 3.12).

\section{Conclusions}

I thank the many of you who used our past solar mixtures, AG89, GN93 and GS98. However, we now advise to forget about these old day solar mixtures and now definitely use the AGSS09 data, refined with the very recent non-LTE results mentioned in Sect. 6, even if they lead to a problem with the Solar Standard Model. This problem first nicknamed "the solar abundance problem" has soon more correctly been recognized as "the solar modeling problem" where, among other fundamental data, the problem of the opacities does play an important role (see Sect. 4, see also Buldgen et al. (2018) and Buldgen et al. (2019) who give some serious hints at the possible solution to this problem). Because of these problems and, maybe, thanks to them, decisive progress is being made in both fields.

\section{Acknowledgements}

We thank Jacques Sauval for many decades of fruitful and friendly collaboration. We also thank Ed Anders, Martin Asplund, Pat Scott and many others who participated in our quest for more accurate solar abundances. Last but not least, I thank Arlette for her more than fifty years of collaboration, friendship and ... love. 
Table 1. Recommended present-day solar photospheric abundances (NEW) compared with the AGSS09 results as well as with the meteoritic data. Values between parentheses are from AGSS09.

\begin{tabular}{llccccc}
$\mathrm{Z}$ & $\mathrm{Elt}$ & $\mathrm{NEW}$ & AGSS09 & NEW-AGSS09 & Met & NEW-M \\
\hline & & & & & & \\
1 & $\mathrm{H}$ & 12 & 12 & - & - & - \\
2 & $\mathrm{He}$ & $(10.93 \pm 0.01)$ & - & - & - & - \\
3 & $\mathrm{Li}$ & $1.07 \pm 0.05$ & 1.05 & 0.02 & 3.25 & -2.18 \\
4 & $\mathrm{Be}$ & $(1.38 \pm 0.09)$ & - & - & 1.32 & 0.06 \\
5 & $\mathrm{~B}$ & $(2.70 \pm 0.30)$ & - & - & 2.78 & 0.08 \\
6 & $\mathrm{C}$ & $8.44 \pm 0.02$ & 8.43 & 0.01 & - & - \\
7 & $\mathrm{~N}$ & $(7.83 \pm 0.05)$ & - & - & - & - \\
8 & $\mathrm{O}$ & $8.69 \pm 0.03$ & 8.69 & 0.00 & - & - \\
9 & $\mathrm{~F}$ & $(4.56 \pm 0.30)$ & - & - & 4.42 & 0.14 \\
10 & $\mathrm{Ne}$ & $8.08 \pm 0.09$ & 7.93 & 0.15 & - & - \\
11 & $\mathrm{Na}$ & $6.21 \pm 0.04$ & 6.24 & -0.03 & 6.26 & -0.05 \\
12 & $\mathrm{Mg}$ & $7.56 \pm 0.04$ & 7.60 & -0.04 & 7.52 & 0.04 \\
13 & $\mathrm{Al}$ & $6.43 \pm 0.03$ & 6.45 & -0.02 & 6.42 & 0.01 \\
14 & $\mathrm{Si}$ & $7.51 \pm 0.03$ & 7.51 & 0.00 & 7.51 & 0.00 \\
15 & $\mathrm{P}$ & $5.41 \pm 0.03$ & 5.41 & 0.00 & 5.44 & -0.03 \\
16 & $\mathrm{~S}$ & $7.12 \pm 0.03$ & 7.12 & 0.00 & 7.15 & -0.03 \\
17 & $\mathrm{Cl}$ & $(5.50 \pm 0.30)$ & - & - & 5.22 & 0.28 \\
18 & $\mathrm{Ar}$ & $(6.40 \pm 0.13)$ & - & - & - & - \\
19 & $\mathrm{~K}$ & $5.07 \pm 0.05$ & 5.03 & 0.04 & 5.08 & -0.01 \\
20 & $\mathrm{Ca}$ & $6.25 \pm 0.09$ & 6.34 & -0.09 & 6.22 & 0.03 \\
21 & $\mathrm{Sc}$ & $3.16 \pm 0.04$ & 3.15 & 0.01 & 3.05 & 0.11 \\
22 & $\mathrm{Ti}$ & $4.90 \pm 0.04$ & 4.95 & -0.05 & 4.91 & -0.01 \\
23 & $\mathrm{~V}$ & $3.89 \pm 0.08$ & 3.93 & -0.04 & 3.95 & -0.06 \\
24 & $\mathrm{Cr}$ & $5.62 \pm 0.04$ & 5.64 & -0.02 & 5.64 & -0.02 \\
25 & $\mathrm{Mn}$ & $5.42 \pm 0.04$ & 5.43 & -0.01 & 5.48 & -0.06 \\
26 & $\mathrm{Fe}$ & $7.48 \pm 0.04$ & 7.50 & -0.02 & 7.47 & 0.01 \\
27 & $\mathrm{Co}$ & $4.93 \pm 0.05$ & 4.99 & -0.06 & 4.87 & 0.06 \\
28 & $\mathrm{Ni}$ & $6.20 \pm 0.04$ & 6.22 & -0.02 & 6.21 & -0.01 \\
29 & $\mathrm{Cu}$ & $4.18 \pm 0.05$ & 4.19 & -0.01 & 4.24 & -0.06 \\
30 & $\mathrm{Zn}$ & $4.56 \pm 0.05$ & 4.56 & 0.00 & 4.61 & -0.05 \\
31 & $\mathrm{Ga}$ & $3.03 \pm 0.05$ & 3.04 & -0.01 & 3.07 & -0.04 \\
32 & $\mathrm{Ge}$ & $3.63 \pm 0.07$ & 3.65 & -0.02 & 3.58 & 0.05 \\
33 & $\mathrm{As}$ & - & - & - & 2.30 & - \\
34 & $\mathrm{Se}$ & - & - & - & 3.34 & - \\
35 & $\mathrm{Br}$ & - & - & - & 2.54 & - \\
36 & $\mathrm{Kr}$ & $(3.25 \pm 0.06)$ & - & - & - & - \\
37 & $\mathrm{Rb}$ & $2.47 \pm 0.107$ & 2.52 & -0.05 & 2.37 & 0.10 \\
38 & $\mathrm{Sr}$ & $2.83 \pm 0.06$ & 2.87 & -0.04 & 2.88 & -0.05 \\
39 & $\mathrm{Y}$ & $2.21 \pm 0.05$ & 2.21 & 0.00 & 2.17 & 0.04 \\
40 & $\mathrm{Zr}$ & $2.59 \pm 0.04$ & 2.58 & 0.01 & 2.52 & 0.07 \\
41 & $\mathrm{Nb}$ & $1.47 \pm 0.06$ & 1.46 & 0.01 & 1.42 & 0.05
\end{tabular}




\begin{tabular}{|c|c|c|c|c|c|c|}
\hline Z & Elt & NEW & AGSS09 & NEW-AGSS09 & Met & NEW-Met \\
\hline 42 & Mo & $1.88 \pm 0.09$ & 1.88 & 0.00 & 1.93 & -0.05 \\
\hline 44 & $\mathrm{Ru}$ & $1.75 \pm 0.08$ & 1.75 & 0.00 & 1.76 & -0.01 \\
\hline 45 & $\mathrm{Rh}$ & $0.89 \pm 0.08$ & 0.91 & -0.02 & 1.06 & -0.17 \\
\hline 46 & $\mathrm{Pd}$ & $1.55 \pm 0.06$ & 1.57 & -0.02 & 1.65 & -0.10 \\
\hline 47 & $\mathrm{Ag}$ & $0.96 \pm 0.10$ & 0.94 & 0.02 & 1.20 & -0.24 \\
\hline 48 & $\mathrm{Cd}$ & $1.77 \pm 0.15$ & - & - & 1.71 & 0.06 \\
\hline 49 & In & $(0.80 \pm 0.20)$ & - & - & 0.76 & 0.04 \\
\hline 50 & Sn & $2.02 \pm 0.10$ & 2.04 & -0.02 & 2.07 & -0.05 \\
\hline 51 & $\mathrm{Sb}$ & - & - & - & 1.01 & - \\
\hline 52 & $\mathrm{Te}$ & - & - & - & 2.18 & - \\
\hline 53 & I & - & - & - & 1.55 & - \\
\hline 54 & $\mathrm{Xe}$ & $(2.24 \pm 0.06)$ & - & - & - & - \\
\hline 55 & Cs & - & - & - & 1.08 & - \\
\hline 56 & $\mathrm{Ba}$ & $2.25 \pm 0.07$ & 2.18 & 0.07 & 2.18 & 0.07 \\
\hline 57 & $\mathrm{La}$ & $1.11 \pm 0.04$ & 1.10 & 0.01 & 1.17 & -0.06 \\
\hline 58 & $\mathrm{Ce}$ & $1.58 \pm 0.04$ & 1.58 & 0.00 & 1.57 & 0.01 \\
\hline 59 & $\operatorname{Pr}$ & $0.72 \pm 0.04$ & 0.72 & 0.00 & 0.75 & -0.03 \\
\hline 60 & $\mathrm{Nd}$ & $1.42 \pm 0.04$ & 1.42 & 0.00 & 1.44 & -0.02 \\
\hline 62 & $\mathrm{Sm}$ & $0.95 \pm 0.04$ & 0.96 & -0.01 & 0.94 & 0.01 \\
\hline 63 & $\mathrm{Eu}$ & $0.52 \pm 0.04$ & 0.52 & 0.00 & 0.52 & 0.00 \\
\hline 64 & $\mathrm{Gd}$ & $1.08 \pm 0.04$ & 1.07 & 0.01 & 1.05 & 0.03 \\
\hline 65 & $\mathrm{~Tb}$ & $0.31 \pm 0.10$ & 0.30 & 0.01 & 0.31 & 0.00 \\
\hline 66 & Dy & $1.10 \pm 0.04$ & 1.10 & 0.00 & 1.13 & -0.03 \\
\hline 67 & Ho & $0.48 \pm 0.11$ & 0.48 & 0.00 & 0.47 & 0.01 \\
\hline 68 & $\mathrm{Er}$ & $0.93 \pm 0.05$ & 0.92 & 0.01 & 0.93 & 0.00 \\
\hline 69 & $\mathrm{Tm}$ & $0.11 \pm 0.04$ & 0.10 & 0.01 & 0.13 & -0.02 \\
\hline 70 & $\mathrm{Yb}$ & $0.85 \pm 0.11$ & 0.84 & 0.01 & 0.92 & -0.07 \\
\hline 71 & $\mathrm{Lu}$ & $0.10 \pm 0.09$ & 0.10 & 0.00 & 0.09 & 0.01 \\
\hline 72 & $\mathrm{Hf}$ & $0.85 \pm 0.05$ & 0.85 & 0.00 & 0.71 & 0.14 \\
\hline 73 & $\mathrm{Ta}$ & - & - & - & -0.16 & - \\
\hline 74 & W & $0.83 \pm 0.11$ & 0.85 & -0.02 & 0.67 & 0.16 \\
\hline 75 & $\operatorname{Re}$ & - & - & - & 0.25 & - \\
\hline 76 & Os & $1.40 \pm 0.05$ & 1.38 & 0.00 & 1.34 & 0.06 \\
\hline 77 & Ir & $1.42 \pm 0.07$ & 1.42 & 0.04 & 1.32 & 0.10 \\
\hline 78 & $\operatorname{Pr}$ & - & - & - & 1.61 & - \\
\hline 79 & $\mathrm{Au}$ & $0.91 \pm 0.08$ & 0.92 & -0.01 & 0.80 & 0.11 \\
\hline 80 & $\mathrm{Hg}$ & - & - & - & 1.17 & - \\
\hline 81 & $\mathrm{Tl}$ & $(0.90 \pm 0.20)$ & - & - & 0.77 & 0.13 \\
\hline 82 & $\mathrm{~Pb}$ & $1.92 \pm 0.08$ & 1.75 & 0.17 & 2.04 & -0.12 \\
\hline 83 & $\mathrm{Bi}$ & - & - & - & 0.04 & - \\
\hline 90 & Th & $0.03 \pm 0.10$ & 0.02 & 0.01 & 0.02 & 0.01 \\
\hline 92 & $\mathrm{U}$ & - & - & - & -0.54 & - \\
\hline
\end{tabular}




\section{References}

Allende Prieto C, Lambert D. L, Asplund M. 2001. ApJ 556, L63

Allende Prieto C, Lambert D. L, Asplund M. 2002. ApJ 573, L137

Amarsi A. M., Asplund M. 2017, MNRAS 464, 264

Amarsi A. M., Barklem P. S., Asplund, M., Collet, R., Zatsarinny, O. 2018, A\&A 616, 89

Amarsi A. M., Barklem P. S., Collet, R., Grevesse, N., Asplund, M. 2019, A\&A 624,111

Anders E., Grevesse N. 1989. GeCoA 53, 197 (AG89)

Asplund M., Grevesse N., Sauval A.J., Allende Prieto C., Kiselman D. 2004, A\&A 417, 751

Asplund M., Grevesse N., Sauval A. J. 2005, ASP Conf. Ser. Barnes TG III, Bash FN, Vol. 336, 25

Asplund M., Grevesse N., Sauval A. J. Scott P., 2009, ARA\&A 47, 481 (AGSS09)

Badnell N. R., Bautista M. A., Butler K., Delahaye F., Mendoza C., Palmeri P., Zeippen C. J., Seaton M. J. 2005, MNRAS 360, 458

Bailey J. E., Nagayama T., Loisel G. P., Rochau G. A., Blancard C., Colgan J., Cosse Ph., Faussurier G., Fontes C. J.; Gilleron F., Golovkin I., Hansen S. B., Iglesias C. A., Kilcrease D. P., Macfarlane J. J., Mancini R. C., Nahar S. N., Orban C., Pain J.-C., Pradhan A. K., Sherrill M., Wilson B. G. 2015, Nature 517, 56

Barrat J. A., Zanda B., Moynier F., Bollinger C., Liorzon C., Bayon G. 2012, GeCoA 83, 79

Basu S., Antia, H. M. 2004, ApJ 606, L85

Bergemann M., Collet R., Amarsi A. M., Kovalev M., Ruchti G. Magic Z. 2017, ApJ 847, 15

Blancard C., Cossé P., Faussurier G. 2012, ApJ 745, 10

Buldgen G., Salmon S., Noels A. 2019, arXiv: 1906.08213

Buldgen G., Salmon S. J. A. J., Noels A., Scuflaire R., Montalbán J., Baturin V.A., Eggenberger P., Gryaznov V. K., Iosilevskiy I. L., Meynet G., Chapli W. J., Miglio A., Oreshina A. V., Richard O., Starostin, A. N. 2018, A\&A 621, 13

Caffau E., Ludwig H.-G., Steffen M., Freytag B., Bonifacio P. 2011, SoPh 268, 255

Carlos M., Melendez J., Spina L., dos Santos L. A., Bedell M., Ramirez I., Asplund M., Bean J. L., Yong D., Yana Galarza J., Alves-Brito A. 2019, MNRAS 485, 405

Christensen-Dalsgaard J., 2002 RvMP 74, 1073

Christensen-Dalsgaard J., di Mauro M. P., Houdek G., Pijpers F. 2009, A\&A 494, 205

Grevesse N., Noels A. 1993, In Origin and Evolution of the Elements, ed. N. Prantzos, E Vangioni-Flam \& M. Cassé, Cambridge, UK, Cambridge Univ. Press., pp 15 (GN93)

Grevesse N, Sauval AJ. 1998. SSRv 85, 161 (GS98)

Grevesse N., Scott P., Asplund M, Sauval A. J. 2015, A\&A 573, 25

Iglesias C A., Rogers F J. 1996, ApJ 464, 943

Krief M., Feigel A., Gazit D. 2016, ApJ 824, 98

Lind K., Amarsi A. M., Asplund M., Barklem P. S., Bautista M., Bergemann M., Collet R., Kiselman D., Leenaarts J., Pereira T. M. D. 2017, MNRAS 468, 4311L

Lodders K., Palme H., Gail H.-P. 2009, Landolt-Börnstein, New Series, Astronomy and Astrophysics, Springer Verlag, Berlin, 71

Nordlander T., Lind K., 2017, A\&A 607, 75

Nordlund A., Stein R. F., Asplund M. 2009, LRSP 6, 2

Osorio Y., Lind K., Barklem P. S., Allende Prieto C., Zatsarinny, O. 2019, A\&A 623, 103

Reggiani H., Amarsi A. M., Lind K., Barklem P. S., Zatsarinny O., Bartschat K., Fursa D. V., Bray I., Spina L., Melendez J. 2019, A\&A 627, 177

Scott P., Grevesse N., Asplund M., Sauval A. J., Lind K., Takeda Y., Collet R., Trampedach R., Hayek, W. 2015a, A\&A,573, 25S

Scott P., Asplund M., Grevesse N., Bergemann M., Sauval A. J. 2015b, A\&A 573, 26S

Serenelli, A. M, Basu, S., Ferguson, J. W., Asplund, M. 2009, ApJ, 705, L123

Stein R. F., Nordlund A. 1998, ApJ 499, 914

Villante F. L. 2010, ApJ 724, 98

Villante, F. L., Serenelli, A. M., Delahaye, F., Pinsonneault, M. H. 2014, ApJ 787, 13

Vinyoles N., Serenelli A.M., Villante F.L., Basu S., Bergström, Gonzalez-Garcia J.M.C., Maltoni M., Peña-Garay C., Song N. ApJ 2017, 835, 202

Young P. R. 2005a, A\&A 439, 361

Young P. R. 2005b, A\&A 444, L45

Young P. R. 2018, ApJ 855, 15 